\title{
ESTRÉS TRAUMÁTICO SECUNDARIO: CÓMO INTERFIERE CON EL DESEMPEÑO DEL PROFESIONAL DE ENFERMERÍA
}

\section{ARTÍCULO ORIGINAL}

COSTA, Sílvia Souza Lima ${ }^{1}$, CAJUEIRO, Laiza Pereira², CARRIJO, Lucilene Santos Pereira $^{3}$, RODRIGUES, Karen Lúcia Abreu ${ }^{4}$

COSTA, Sílvia Souza Lima. Et al. Estrés traumático secundario: cómo interfiere con el desempeño del profesional de enfermería. Revista Científica Multidisciplinar Núcleo do Conhecimento. Año 06, Ed. 01, Vol. 07, págs. 38-56. Enero de 2021. ISSN: 2448-0959, Enlace de acceso: https://www.nucleodoconhecimento.com.br/salud/estres-traumatico-secundario, DOI: 10.32749/nucleodoconhecimento.com.br/salud/estres-traumatico-secundario

\section{RESUMEN}

El desempeño del profesional de enfermería sufre la interferencia del Estrés Traumático Secundario (ETS), estrés laboral que afecta a los profesionales de la salud. Este trabajo tiene como objetivo comprender las implicaciones del ETS en la vida profesional, sus formas de prevención y cómo intervenir, con el fin de promover la calidad de vida laboral, lo que implica la salud física y mental. Este estudio se llevó a cabo mediante revisión bibliográfica de literatura, a partir del análisis de artículos y selección de libros de texto en el campo de la Psiquiatría, con el objetivo de solidificar el tema y los artículos científicos en la base de datos de la biblioteca virtual: Google Scholar, Pubmed, Scielo. ¿En qué medida el uso de la empatía interfiere en la vida diaria del profesional? Las profesiones auxiliares sufren el efecto de aplicar

\footnotetext{
${ }^{1}$ Académica del curso de Enfermería de la Faculdade Morgana Potrich (FAMP), Mineiros - GO, Brasil.

${ }^{2}$ Académica del curso de Enfermería de la Faculdade Morgana Potrich (FAMP), Mineiros - GO, Brasil.

${ }^{3}$ Académico del curso de Nutrición de la Faculdade Morgana Potrich (FAMP), Mineiros - GO, Brasil.

${ }^{4}$ Tutor. Profa. Esp. Psicología por el Morgana Potrich College (FAMP), Mineiros-GO. Profesor de la FAMP Faculdade Morgana Potrich, Mineiros-GO. Brasil, Licenciatura en Psicología en UFMT- Universidad Federal de Mato Grosso, especialización en Gestión Organizacional, Desarrollo Humano y Coaching en Avilá Pós-Goiânia, Gestión de Aula en Educación Superior en Unifimes - Mineiros.
}

RC: 79470

Disponible: https://www.nucleodoconhecimento.com.br/salud/estres-traumaticosecundario 
sentimientos una reacción espontánea que se produce al final de la vivencia de un hecho vivido por otro. Tiene fama de fatiga, resultante del estrés laboral en su fase de agotamiento, que conduce a la anestesia emocional. Los estudios muestran que la reacción del ETS se vuelve acumulativa, perjudicando la atención brindada, generando cambios cognitivos, afectivos y relacionales, produciendo miedo, tristeza y depresión. La literatura es escasa sobre estudios relacionados con esta temática en Enfermería, y es fundamental desarrollar estudios para esclarecer aún más el tema. El conocimiento y el seguimiento sobre el Estrés Traumático Secundario aportan actitudes preventivas, como medio para promover la calidad de vida, basadas en el principio de que la prevención sigue siendo la mejor medicina para no enfermarse.

Palabras clave: Estrés, estrés traumático, Burnout, fatiga compasiva.

\section{INTRODUCCIÓN}

El Estrés Traumático Secundario (ETS) afecta las profesiones de ayudar a otros en peligro, resulta de la exposición prolongada al dolor ajeno y se configura como una reacción espontánea experimentada por el profesional después de que el otro atraviesa una experiencia estresante; proviene del estrés en su fase aguda, del agotamiento emocional, que acaba acarreando pérdidas en la vida del profesional (CASTRO, 2018).

El estrés se entiende como una respuesta fisiológica del organismo ante situaciones que llevan al organismo a buscar cambios internos, requiriendo una rápida respuesta individual en el lugar donde se inserta. Una adaptación que te prepara para afrontar la agitación cambiando tu comportamiento, con el fin de restablecer un control interno de tu equilibrio (TRETTENE, 2016).

El área de la salud es un lugar propicio para el desarrollo de enfermedades en los profesionales debido al estrés, desencadenando reacciones en el organismo y favoreciendo la adaptación momentánea. Produciendo así un aumento de adrenalina, 
generando energía en el primer momento y estimulando un mayor rendimiento (VAZATA, 2015).

La larga duración del estrés conduce a daños físicos, llegando al agotamiento físico y psicológico, a una caída en la realización de los cuidados y en la propia salud del trabajador, mostrando una sensación de agotamiento, cansancio, como el Síndrome de Burnout, si el individuo no sabe cómo para gestionarlo (MORAES, 2016).

El trabajo es una parte indispensable de la vida humana. Es a través de él que el hombre puede vivir e interactuar en la sociedad de producción. Sin embargo, los cambios que se han producido en las últimas décadas han provocado cambios en el entorno laboral y, dependiendo de cómo se realice el trabajo, estará directamente relacionado con los factores y determinantes del desgaste durante el proceso de la enfermedad (TEIXEIRA, 2019).

La preocupación por la calidad de vida en el trabajo surgió alrededor de la década de 1960, cuando se dio cuenta de que los trabajadores más felices y motivados producían más y mejor. Además de incentivar buenos salarios y beneficios atractivos, la calidad de vida en el trabajo consiste en un conjunto de técnicas y actividades orientadas a la satisfacción de los empleados (SANCHEZ, 2019).

Cuando los profesionales de la salud desempeñan sus funciones a través de la empatía (comprensión emocional e identificación con otra persona), tienen niveles de compasión que pueden llevarlos a sentir miedo, similar al dolor y sufrimiento de los demás. Esta situación genera fatiga por compasión, un arduo agotamiento biológico que impide que el organismo libere energía psíquica por el uso excesivo de sentimientos de compasión, dando lugar al Trastorno de Estrés Traumático Secundario (TEIXEIRA, 2019).

Considerando las consecuencias que pueden desencadenar el estrés, se observa la importancia de detectar los signos y síntomas en su etapa inicial, interfiriendo para prevenir su evolución, ya que los síntomas pueden causar daño a la salud del profesional. Así, se vuelve imprescindible conocer, detectar, vigilar la condición e 
intervenir para que no evolucione hacia el agotamiento emocional, que conduce al Trastorno de Estrés Traumático Secundario.

Por tanto, este estudio tiene como objetivo comprender los factores que provocan el Estrés Traumático Secundario (ETS) en los profesionales de enfermería, sus reacciones y qué se puede hacer en prevención. Reconocer su influencia, comprender los principales factores que contribuyen e identificar las implicaciones que se derivan de ella; el valor en prevención, conociendo el ICD relacionado con ETS y su notificación; ofreciendo alternativas laborales que contribuyan a la calidad de vida del profesional.

\section{METODOLOGÍA}

El presente trabajo explica la revisión bibliográfica, un método de investigación capaz de favorecer la asociación de conocimientos y la integración de saberes expresivos para la comprensión de un tema o fenómeno. (VIEIRA, 2018).

El primer paso se dio a través de la elaboración de la pregunta orientadora del trabajo; en el segundo, se buscaron en la base de datos y descriptores temas directamente relacionados con el tema. Se observó los resúmenes de los artículos involucrados en la investigación, seleccionados aquellos artículos relacionados con el tema. Finalmente, se cruzaron los descriptores de palabras clave: Estrés. Estrés secundario. Burnout. Fatiga de la compasión. Estrés post traumático.

Así, como medio para investigar el tema y solidificarlo, se incluyeron en el trabajo libros de texto del área de Psicología y Psiquiatría.

Por tanto, la revisión de los artículos se realizó entre enero y marzo de 2020. Para identificar y seleccionar los artículos se utilizó la base de datos electrónica Scielo, Google Scholar, Pubmed, Bases Bireme, Biblioteca Virtual de Salud (BVS), definiendo luego los descriptores e insertando ellos en la búsqueda de la inclusión. Los términos utilizados se separaron y se utilizaron en la búsqueda de descriptores. A partir de las palabras clave de los artículos relacionados con el tema, se hizo efectivo priorizar los 
artículos brasileños, para conocer la implicación académica en el tema en cuestión. Y como forma de involucrar al contexto histórico, se incluyó un artículo con la fecha además de los cinco años de publicación por su valor histórico.

\section{ESTRÉS}

El estrés es un proceso interno del organismo que requiere una resolución multifacética del sistema corporal que incluye cambios en el comportamiento humano. $Y$ surge del evento momentáneo que requiere reacciones cognitivas, emocionales para adaptarse al entorno, a fin de mantener el equilibrio interno ante la amenaza momentánea; cada individuo responde a estímulos con características propias, generando su propio estrés (COSTA, 2016).

Los profesionales de enfermería calificados generalmente brindan servicios específicos para el cuidado de la población frágil o enferma, que necesita asistencia médica hospitalaria. Por tanto, ante esta demanda, los especialistas consumen energía física, propulsando al organismo a cambios internos, drenando las reservas funcionales mediante la quema de energía, buscando adaptarse al entorno produciendo estrés (VAZATA, 2015).

Los trabajadores que trabajan en lugares que requieren una acción rápida, conocimiento científico técnico y una conducta rigurosa, como en los hospitales, tienen altos niveles de estrés (PRETO, 2016).

Las tareas que se deben realizar en poco tiempo, ruido en el ambiente, entre otros aspectos considerados causantes de estrés pueden generar síntomas como irritación, pérdida de atención, sensación de agotamiento, dolor de cabeza, dolores musculares, cambios en la presión arterial y latidos cardíacos; estos cambios tienden a favorecer la evolución del estrés en los profesionales de enfermería y terminan empeorando la mala calidad del sueño, lo que produce más desgaste que satisfacción (VERSA, 2012; VAZATA, 2015). 
Así, quienes logran satisfacción en el ambiente laboral tienden a tener un índice de estrés bajo, evitando problemas de salud física y psicológica, mientras que la homeostasis afectada por la gran cantidad de estrés genera tensiones, que pueden resultar en el desgaste del cuerpo provocando agotamiento. comprometiendo así la salud del colaborador del equipo (GOMES, 2017).

Hans Selye es considerado el pionero en mencionar el estrés biológico. En 1925, como estudiante de Medicina se identificó al recibir a la población con diversas patologías en la clínica. Los signos y síntomas como la delgadez, la falta de apetito y el tono muscular disminuyeron, ya que las respuestas eran comunes en personas sometidas a la hostilidad. Así, deliberó como una "reacción defensiva fisiológica del sistema ante cualquier estímulo", dividiéndolo en tres fases: reacción de alarma, resistencia y agotamiento (SILVA, 2018).

\subsection{FASES DE ESTRÉS}

En la fase de alarma, el estrés parece insignificante, sin embargo, el cuerpo proporciona más energía para quemar con el fin de mantener la homeostasis interna. La frecuencia cardíaca aumenta, aumenta la sudoración, se producen tensión muscular y nerviosismo, como respuestas a los agresores (SILVA, 2018; VAZATA, 2015).

La segunda fase es la fase de resistencia, en la que el cuerpo demanda cambios internos a los estresores, consumiendo reservas orgánicas para mantenerse adaptado a la nueva situación. Presentando así signos como inquietud, soledad, aprensión, falta de apetito o glotonería; si los estresores son continuos, el organismo debilitado pasa a la fase de agotamiento, la tercera fase, en la que las enfermedades se manifiestan en problemas cardíacos, respiratorios, trastornos gastrointestinales, porque el organismo no se puede adaptar (SILVA, 2018).

Para Vazata (2015), el agotamiento es una "ruptura total de la resistencia", que genera enfermedades. De esta forma, los sujetos que luchan internamente pueden desarrollar 
el proceso de afrontamiento, utilizando el mecanismo de escape como consecuencia de la fragilidad del organismo.

\subsection{EMPATÍA}

Según Terezam (2017), la empatía es la capacidad de posicionarse en el lugar de las personas, con el fin de idealizar y vivir en una misma concepción la experiencia vivida por los demás. Comportamiento importante para la comodidad, seguridad, tranquilidad física y mental de ambas partes involucradas. Conciencia de los propios miedos, asimilación de emociones y sentimientos declarados por el paciente favoreciendo una relación empática.

Según Savieto (2016), la empatía se establece como la eficacia de analizar los sentimientos ajenos. Ponte en la situación de cada persona, comprende sus concepciones y emociones. Así, los profesionales de la salud como enfermeras con habilidades empáticas pueden comprender al paciente y establecer asistencia para mejorar su vida.

Sin embargo, el equipo de enfermería debe mantener un alto nivel de sabiduría, persistencia emocional y comprensión, para que la empatía emerja como un proceso terapéutico. $Y$ es fundamental que se haga con anticipación una conexión de respeto mutuo y que el enfermero realice cuidados individualizados, respetando la etnia, creencias y moral del cliente (ALBUQUERQUE, 2019).

Según Gambarelli (2018), a través de la asistencia experimentada entre la enfermera y el paciente, este especialista puede determinar los objetivos y propósitos a alcanzar para el cliente, por sí mismo o en equipo, con el fin de acomodar al paciente y estar en conformidad. con ser un ser humano integral, capaz de lograr resultados por sus dificultades. El paciente debe aceptar de estos especialistas lo esencial para el progreso y rehabilitación de su salud. 


\subsection{SÍNDROME DE QUEMADURA}

El Burnout es un proceso gradual seguido de negativas durante el ejercicio de la práctica profesional, generando apatía, insensibilidad, falta de actividad y reacción, derivado del estrés frecuente en el trabajo. Entonces, las actitudes negativas hacia los clientes comienzan como una defensa, dejando el cariño y comenzando a actuar de forma lógica racional, para no ser golpeados por estresores (OLIVEIRA; RODRIGUES; SANTANA, 2017).

El síndrome de Burnout indica agotamiento de la mente en relación con el ejercicio. Es una sensación de fracaso y agotamiento por el desgaste excesivo de los profesionales. Se genera en la implicación con las personas y provocada por el trabajo, lo que hace que el profesional se desanime, con bajo rendimiento, falta de autoestima y decadencia profesional. Porque se sienten impotentes y sin placer en hacer su trabajo (BENEDITO, 2017).

Dado que el contacto continuo y directo con el sufrimiento de la muerte de los pacientes requiere del profesional un alto conocimiento sobre sus capacidades. Además de ser muy exigente en el ámbito hospitalario a la hora de tomar decisiones, también influye directamente en la vida de los demás. Esta carga de responsabilidad genera reacciones y síntomas individuales, derivados de los niveles de presión y estrés existentes en el entorno. En última instancia, conduce a un estado de agotamiento físico y mental. (FREITAS, 2017).

En el Síndrome de Burnout el profesional atraviesa experiencias de agotamiento físico y emocional, debido al agotamiento provocado por el ejercicio profesional. Además de la debilidad física y las reacciones emocionales, que derivan en sentimientos de falta de preparación y conductas negativas en el entorno laboral, alejamiento de los clientes, enfermedad de los profesionales que lo acompañan, crecimiento del absentismo y aumento de los costos de salud para la empresa, que pueden generar la decisión para cambiar de profesión para quienes pasan por el Síndrome. (RUBACK, 2018).

RC: 79470

Disponible: https://www.nucleodoconhecimento.com.br/salud/estres-traumaticosecundario 


\subsection{ESTRÉS TRAUMÁTICO SECUNDARIO}

El Estrés Traumático Secundario (ETS) surge del cuidado de individuos en angustia, resultado del estrés en su fase de agotamiento emocional, generado por la vivencia de situaciones que vive otra persona y el profesional participa en el cuidado. Produciendo así síntomas similares a los del enfermo, con repercusiones en su propio desempeño y sentimiento (CASTRO, 2018).

Sin embargo, una reacción espontánea que se produce en el profesional de la ayuda (enfermeras, médicos, bomberos, policía) derivada del desenlace de la experiencia sobre un hecho vivido por otra persona. Por ejemplo, un trauma indirecto, con efecto acumulativo en la prestación de ayuda o asistencia, provocando retroalimentación, respuesta de miedo, impotencia u horror (VALENTE, 2013).

El Estrés Traumático Secundario también puede indicar "fatiga compasiva" relacionada con la destrucción progresiva de la empatía que es capaz de llegar a los especialistas en salud. Al inicio de sus profesiones, estos especialistas tienen un alto grado de empatía. Sin embargo, puede reducirse por el estrés diario provocado por el continuo despliegue del sufrimiento de los pacientes (TEREZAM, 2016). EI ETS tiene sinónimos como "tensión traumática secundaria, traumatización secundaria, traumatización indirecta, superviviente secundario, contagio emocional, fatiga compasiva" (RUIZ, 2017).

EI ETS afecta a un grupo de cuidadores profesionales que se ocupan del sufrimiento ajeno, como: riesgo de muerte, enfermos o víctimas de hechos traumáticos. Porque pueden contagiarse del dolor real del otro, llegando al agotamiento por compasión resultante de la sensibilidad y el cuidado de los demás. Generando así, con el tiempo, una merma en su capacidad de experimentar alegría o de sentir preocupación por alguien (BARBOSA, 2014).

Sin embargo, ETS se genera por la implicación del profesional que experimenta el dolor ajeno. Esta actuación produce síntomas de Trastorno de Estrés Postraumático 
(TEPT), similar al del paciente, resultado del intento de ayudar al ser humano que sufre, impactando negativamente en el psíquico cognitivo del profesional (CASTRO, 2018).

La participación de enfermeras profesionales durante su jornada laboral las coloca frente a cargas biopsicológicas. Y la forma en que cada individuo reacciona a estas tensiones puede producir una disminución de su productividad, provocando enfermedad y absentismo laboral (FELLI, 2015).

\subsection{SÍNTOMAS ESTRÉS TRAUMÁTICO SECUNDARIO}

El síntoma de Estrés Traumático Secundario surge luego de que el profesional de la salud atiende a personas debilitadas por los sentimientos de empatía involucrados en el cuidado brindado, en las cuales comienzan a sentir el dolor ajeno aún sin haber sufrido el daño. Este sentimiento se llama estrés traumático secundario, porque cambia la homeostasis interna del profesional. Sin embargo, los primeros análisis de este sentimiento tuvieron lugar en los familiares de los veteranos de guerra, en 1974, cuando los familiares involucrados sintieron los síntomas a pesar de no estar realizando la guerra. Sin embargo, se entendió que la experiencia traumática en personas sanas puede desarrollar reacciones sintomáticas al TEPT luego de experimentar cierto hecho (APOIAR, 2017).

De acuerdo con la 5ta edición del Manual Diagnóstico y Estadístico de los Trastornos Mentales (DSM-5), los eventos traumáticos desencadenan a las personas involucradas o cercanas, experimentando el evento. Reacciones de evitación, excitabilidad, cambios negativos en el estado de ánimo y cognición, que producen angustia psicológica, caída en la vida social y profesional, conocidos como Traumatismos y Trastornos Relacionados con el Estrés (ARAÚJO, 2014).

Es necesario que los síntomas presentados estén vinculados al episodio traumático estresante, vivido, presenciado o confrontado ante uno o más factores que involucran la muerte, lesiones graves reales o miedo en su plenitud en la persona debida u otra 
persona. Tales como la pérdida de un miembro del cuerpo en accidentes automovilísticos o en el trabajo, violación, tortura física, bandidaje violento, explosiones y eventos naturales; inundaciones, ataques de animales, terremotos, erupciones volcánicas. Estas situaciones conducen al miedo, la incredulidad, la desesperación, la decepción (APOIAR, 2017).

\subsubsection{CAMBIOS EN EL PROFESIONAL DERIVADOS DEL ESTRÉS TRAUMÁTICO SECUNDARIO}

Durante la ejecución y desarrollo del ejercicio de Enfermería, el cuerpo del profesional sufre cambios relacionados con el estrés y en cada fase del mismo, lo que se conoce como Estrés Laboral. Este tipo de estrés genera manifestaciones psicológicas en cada individuo debido al estrés en su fase de agotamiento. Según Fabri, el 50\% de los profesionales expresan deseo de evasión, $50 \%$ de agonía y aprensión diaria, además de un $45 \%$ que reporta fatiga demodulada que conduce a la fragilidad del organismo (FABRI, 2018).

Como resultado, el sufrimiento causa una reacción biológica disfuncional en el cuerpo, como resultado de la exposición prolongada al dolor de otros. La llamada fatiga compasiva, producto de la empatía, la responsabilidad por el paciente y su sufrimiento, un desvanecimiento crónico del cuidado y la preocupación por el otro (SANTANA, 2017).

Los hechos traumáticos transforman sensaciones en el profesional tras la experiencia, generan miedo y trauma, alteran lo cognitivo, modifican la respuesta y reacciones fisiológicas. Los recuerdos del trauma inducen miedo, el cual lleva a escapar, como protección frente a un estresante, para mantener una homeostasis, ya que el trauma genera ansiedad (SBARDELLOTO, 2012).

Así, los cambios cognitivos aparecen como pensamientos intrusivos, hipervigilancia, dificultades de concentración, pérdida de sueño, ideas fijas y sobrevaloradas de peligro en la sociedad. Como recuerdos de imágenes desagradables, entre otras. 
Todo esto acaba desencadenando preocupaciones excesivas por la familia y desesperanza en su vida diaria. A nivel afectivo produce miedo, ansiedad, malestar, irritabilidad, enfado, tristeza, melancolía, culpa e insensibilidad. Y a nivel fisiológico, náuseas, fatiga, escalofríos, dolor de cabeza, taquicardia, dolor de cabeza, trastornos digestivos (RUIZ, 2017).

Por tanto, los síntomas de origen psicótico pueden aparecer en profesionales con la mente agotada. Reflejándose en la vida diaria como la sensación de cansancio, tristeza, desánimo, disminuyendo así la capacidad cognitiva, interfiriendo en la calidad del sueño y produciendo ansiedad, que se incrementan durante el trabajo diario (ALVES, 2015).

\subsection{SEGUIMIENTO PROFESIONAL}

EI SISMOTE (Sistema de Monitoreo del Trabajador de Enfermería) es un sistema que fue desarrollado y aprobado para comprender los problemas de salud en los profesionales de la salud, mediante indicadores. Con el fin de presentar el perfil de fragilidad de los trabajadores y posibilitar la planificación de intervenciones para minimizar las circunstancias de episodios laborales degradantes (FELLI, 2015; GUIMARÃES, 2016).

CEREST (Centro de Referencia en Salud Ocupacional) es una agencia que opera desde 2004. Especialista en el cuidado de la vitalidad del empleado, utiliza el modelo de Atención Básica, conectado a la Red Nacional de Atención Integral a la Salud Ocupacional - RENAST, analizando los actores que implican la salud de los trabajadores (CEREST, 2015).

Así, CEREST tiene como objetivo minimizar los accidentes y lesiones derivados del trabajo en la vida del profesional, mediante intervenciones en el área de desarrollo. Como recuperación, precaución en el ámbito de la salud, contiene información sobre la salud del trabajador y se estructura con acciones de empoderamiento de los empleados. Tiene a RENAST como una de las tácticas para certificar la atención 
integral de salud a los trabajadores, como uno de los lineamientos de la Política Nacional de Salud Ocupacional del Ministerio de Salud. Presentando, entonces, el propósito de constituir la red de servicios del SUS, impulsada por la cooperación y observación de prácticas de Salud Ocupacional a través de la prevención y vigilancia (CEREST, 2015).

Los cambios en el profesional provocados por el estrés en el día a día de la profesión que conducen a un estrés laboral que da lugar a un estrés secundario se pueden clasificar según la Clasificación Internacional de Enfermedades: CIE-10, en CIE F.43, por presentar síntomas. similar al estrés postraumático (PTSD). Esta estandarización de signos y síntomas a través de la CIE es universal, facilitando su seguimiento a través de la estandarización (CIE 10, 1996).

Por tanto, el agotamiento del profesional puede derivar en inestabilidad psicológica, provocando trastorno mental, perjudicando el desempeño de tareas en su vida diaria. La literatura sobre los trastornos de enfermería es escasa y, según Alves, los trastornos mentales constituyen el $12 \%$ de las discapacidades en la humanidad, lo que dificulta su reconocimiento temprano por su carácter indefinido (ALVES, 2015).

\subsection{CALIDAD DE VIDA LABORAL}

Para favorecer el desarrollo de una calidad de vida satisfactoria, el entorno laboral necesita ofrecer apoyo social, seguridad, un buen salario, carga de trabajo adecuada, reconocimiento de los profesionales y oportunidades de crecimiento. La calidad de vida en el trabajo se define como un aspecto importante en la decisión del enfermero de permanecer en el puesto de trabajo, manteniéndose calificado, satisfecho y productivo (HIPÓLITO, 2016).

La calidad de vida en el trabajo está relacionada con una serie de acciones que lleva a cabo la empresa que deben brindar condiciones laborales adecuadas. Como desarrollo humano, buenas habilidades interpersonales y felicidad dentro y fuera de la empresa. Así, en el entendido de que esto ayuda a incrementar la productividad de 
los empleados, brinda disposición y motivación a través de las cuales los empleados pueden lograr resultados notables (SANCHEZ, 2019).

Si bien la falta de motivación, el alto estrés y el ambiente laboral desagradable son algunas de las principales razones de la mala calidad de vida en la empresa. Como resultado, la organización puede sufrir altas tasas de absentismo y bajas por enfermedad. Con la aplicación de los principios de calidad de vida en el trabajo, los empleados se beneficiarán de condiciones laborales más saludables, además de la debida atención a su salud y seguridad, y el beneficio es la mejora de la salud física, mental, emocional y la reducción de enfermedades (HIPÓLITO, 2016).

\subsection{LA ENFERMERA}

El enfermero es responsable de articular las acciones y sus decisiones con el fin de utilizar la tecnología a favor del bienestar del cliente, destacándose como líder en la asistencia brindada por el equipo, a través de la gestión e involucrando sus prácticas y conocimientos adquiridos en su formación. , calificación y problemas de salud, produciendo promoción y recuperación de la salud (COFEN, 2020).

Se destaca la actuación de la Enfermería desde el Servicio Móvil Terrestre y Acuático Prehospitalario, la atención directa y el Centro de Regulación de Emergencias, minimizando los riesgos de enfermedades, en el ahorro en la administración de medicamentos, en el transporte de pacientes, en la sistematización de la asistencia a través de la implementación del proceso de enfermería (COFEN, 2020).

El área de Enfermería es de suma importancia para el paciente y la sociedad, con el objetivo de garantizar y ofrecer el derecho a la salud y la vida. En este contexto, es necesario reconocer que toda persona tiene derecho a una adecuada atención de enfermería, de manera humanizada, integrada y solidaria (FROTA, 2019).

La Enfermera se alía con la calidad de la atención brindada gracias a la implicación con los pacientes y familiares. La profesión forma parte de un eje de suma importancia 
en el proceso de enfermedad y hospitalización, donde el vínculo que se crea es de confianza, ayudando en los casos de debilidad de los pacientes (FROTA, 2019).

El enfermero puede verse influenciado por el sufrimiento ajeno, llegando al agotamiento por compasión resultante del desempeño profesional en el cuidado. Generando así, con el tiempo, disminución de la empatía y experimentar alegría o sentimiento de preocupación por alguien (BARBOSA, 2014).

Al atender a personas frágiles, el contacto con el dolor ajeno, puede desencadenar Estrés Traumático Secundario en la Enfermera profesional a lo largo de los años (CASTRO, 2018).

\section{RESULTADOS Y DISCUSIÓN}

ETS proviene del estrés en su fase de agotamiento emocional, resultado de los sentimientos que envuelven la empatía y la compasión. Asisten personas que se encuentran frágiles en momentos de dolor, que pueden ser accidentes o torturas, eventos naturales (inundaciones, terremotos, erupción volcánica), situaciones que brindan miedo, impotencia y desesperación (RISSARDO, 2013; APOIAR, 2017).

Según Ruiz (2017), la ETS representa las emociones que emergen en el profesional luego de brindar atención a personas que han pasado por situaciones dolorosas, resultado de la empatía en la interacción profesional, en la que el profesional atraviesa cambios en el cuerpo que conducen a emociones. agotamiento y está vinculado al estrés laboral.

Sin embargo, los factores que provocan el Estrés Traumático Secundario en los profesionales de Enfermería provienen del desgaste psicológico explícito de las funcionalidades orgánicas del ser humano, cuya implicación del desempeño técnico junto con la condición del paciente sumado a la doble jornada laboral, familiar, rutinaria y funciones que requieren compromiso corporal y psíquico predisponen al trabajador de la salud al ETS (SANTANA, 2017). 
El acto de negar síntomas se refiere a un rechazo consciente a percibir hechos perturbadores, lo que genera conductas defensivas, entre ellas el aislamiento, el rechazo. Generando así perturbaciones en las relaciones que se establecen en el trabajo, que pueden dañar todos los ámbitos de la vida del profesional (FREIRE, 2017).

Sin embargo, en el trabajo, cada profesional tiene su individualidad y modo de adaptación en el entorno insertado, cuyas reacciones pueden conducir a futuras enfermedades en función de la fase de estrés. Porque, el organismo no puede adaptarse a la demanda de reacciones internas y emocionales reales en el lugar de ocupación o experiencia (GOMES, 2017).

El Burnout está relacionado con la exigencia de responsabilidad en el trabajo, asociado a la complejidad de desempeñar funciones dentro de medios estresados, sin aliento y desfavorecidos (APOIAR, 2017). Afecta a los empleados que tratan con personas y actividades relacionadas con el público, y se da por la dinámica del trabajo (RODRIGUES; SANTANA, 2017).

Algunas mujeres suelen mostrar sus emociones con mayor facilidad, por lo que toman precauciones para obtener ayuda emocional. Los hombres, en cambio, son todo lo contrario, suelen aceptar poco cuidado. Esta eficiencia de las mujeres en dar mayor valor a las sensaciones es un proceso fundamental para las enfermeras. Lo que otorga una comprensión de los sentimientos, promoviendo una gran cooperación de la condición del paciente, que se vincula con la empatía (MUFATO, 2019)

Sin embargo, el sentimiento ajeno se ve disminuido, lo que se conoce como anestesia emocional, atenuación de la disposición o actividades que alguna vez fueron placenteras, como una caída en la implicación que antes le traía compasión y bondad a uno mismo. (VALENTE, 2013).

En Brasil, el fenómeno ETS es poco conocido. Así, basando los estudios en el trabajo de Kennyston Lago y Wanderley, bajo la mirada de la enfermería (SANTANA, 2014), 
existen trabajos directamente vinculados al tema en otros idiomas, como el inglés y el español (LORENZO, 2017).

Ruiz (2017) afirma que el Estrés Traumático Secundario es un tema nuevo que tiene el potencial de interferir con el desempeño de las tareas diarias durante la jornada laboral e influye en el desarrollo de condiciones depresivas. Modificando así las reacciones cognitivas, fisiológicas y su propia conducta. Desarrollando entonces, la evitación como medio de protección en tu subconsciente, provocando desmotivación de actividades previamente placenteras y aumentando la sensación de agotamiento, pues como medio para tratar de evitar lo vivido, te dedicas a trabajar sin cesar.

Según Teixeira, el estrés indica imposición, haciendo que el profesional de enfermería se desvanezca, influyendo en la homeostasis de su cuerpo, debido al enorme cúmulo de tensiones que se da en la vida cotidiana (TEIXEIRA, 2019).

La empatía es una calificación que se puede ejercer ante el paciente de forma oral y no oral. Y su progresión es de gran importancia, porque es a través de ella que estamos dispuestos a colaborar con las personas (ALBUQUERQUE, 2019).

Según Guimarães (2016), existe dificultad para monitorear la salud de las enfermeras debido a las notas insuficientes en las organizaciones sobre los daños procesados. Monitorear el bienestar y la vitalidad del personal de enfermería es un gran desafío. Dado que los trastornos mentales desarrollados por la realización del trabajo fueron reportados como situaciones de depresión y ansiedad, derivadas del agotamiento emocional, representando un pedido de la psique del trabajador que no contó con apoyo grupal. Su evitación es posible mediante medidas preventivas y de protección.

Así, el apoyo psicológico y el apoyo social, según Ruiz (2017), son fundamentales para que los profesionales puedan afrontar situaciones de emergencia sin desarrollar ETS. Porque la carga de trabajo sin apoyo tiene un efecto negativo en todas las demás áreas de la vida del profesional, disminuyendo la capacidad de afrontamiento. 
La psicoterapia es un medio de atención en el que, a través de la comunicación verbal, el diálogo, es posible verificar la queja del profesional y los problemas relacionados con su profesión, interfiriendo de manera que se modifique el problema causado por las emociones, la conducta y la cognición. Protegiendo así tu emocional, ayudando a fortalecer las reacciones, a través del diálogo entre los profesionales y el enfermero que gestiona el lugar, con el fin de encontrar formas específicas de mantener el equilibrio emocional, reduciendo los riesgos de producir enfermedades a través del estrés (GUIMARÃES, 2016).

\section{CONSIDERACIONES FINALES}

Respetar el límite del cuerpo, conocer sus debilidades puede contribuir a la reducción del estrés, proporcionando satisfacción durante el desempeño del profesional y evitando el desgaste en la fase de agotamiento que genera cambios en el cuerpo y la mente, modificando la sensación involucrada en el ejecución, la empatía, dando lugar a ETS. Ofrecer las condiciones psíquicas profesionales favorables para el cuidado saludable de uno mismo, entonces, produce una asistencia calificada, ya que el enfermero es también un ser humano con debilidades que pueden interferir en su profesión diaria; Las sobrecargas diarias pueden dañar la calidad de la atención brindada, aumentando el estrés diario y perjudicando la calidad de vida.

Así, para que el profesional tenga una buena salud física o mental, debe existir un ambiente agradable, que a su vez proporcione una buena calidad de vida, reduciendo los factores desencadenantes del estrés que promueven el Estrés Traumático Secundario en los profesionales de Enfermería.

Se concluye con esta investigación que el estrés en la fase de agotamiento proporciona la aparición del Síndrome de Burnout y Estrés Traumático Secundario, haciendo importante el seguimiento del profesional, ya que contribuye significativamente a las intervenciones relacionadas con las debilidades individuales, con el fin de reducir el estrés oxidativo, contribuyendo así a una mejor calidad de vida ya un profesional que ofrece asistencia calificada. 


\section{REFERENCIAS}

ALBUQUERQUE, M. C.S. et al., NURSES' EMPATHY IN AN EMERGENCY HOSPITAL SERVICE. Texto context - enferm., Florianópolis, v. 28, e20170406, 2019. Disponível em: <http://www.scielo.br/scielo.php?script=sci_arttext\&pid=S0104$07072019000100327 \&$ Ing=en\&nrm=iso >. Acesso em 03 mar. 2020.

ALVES, A. P. et al. Prevalência de transtornos mentais comuns entre profissionais de saúde [Prevalence of common mental disorders among health professionals]. Revista Enfermagem UERJ, [S.I.], v. 23, n. 1, p. 64-69, mar. 2015. ISSN 0104-3552. Disponível em:

$<$ https://www.epublicacoes.uerj.br/index.php/enfermagemuerj/article/view/8150/12330>. Acesso em: 08 ago. 2020.

APOIAR, ASSOCIAÇÃO DE APOIO AOS EX-COMBATENTES VÍTIMAS DO STRESS DE GUERRA. Artigo Científico. Conceito de Traumatização Secundária. Faculdade de Psicologia e Ciências da Educação da Universidade de Lisboa. 19, set. 2017. Disponível em: <https://apoiarstressdeguerra.com/pt/2013/04/01/tese-de-mestrado-em-perturbacao-secundaria-destress-traumatico-stsd/>. Acesso em: 26 fev. 2020.

ARAUJO, A. C.; LOTUFO NETO, F.; A nova classificação Americana para os Transtornos Mentais: o DSM-5. Rev. bras. ter. comport. cogn., São Paulo, v. 16, n. 1, p. 67-82, abr. $2014 . \quad$ disponível em <http://pepsic.bvsalud.org/scielo.php?script=sci_arttext\&pid=S151755452014000100007\&lng=pt\&nrm=iso >. Acessos em 24 fev. 2020.

BARBOSA, S.C; SOUZA, S.; MOREIRA, J.S.; A fadiga por compaixão como ameaça à qualidade de vida profissional em prestadores de serviços hospitalares. Rev. Psicol., Organ. Trab., Florianópolis, v. 14, n. 3, p. 315-323, set. 2014. Disponível em: <http://pepsic.bvsalud.org/scielo.php?script=sci_arttext\&pid=S198466572014000300007\&lng=pt\&nrm=iso >. Acesso em: 11 jan. 2020. 
BARRETO, J.B.M; CAMPOS, C.A.; A psicoterapia de apoio como método para atender à demanda de queixa nos relacionamentos interpessoais: um estudo de caso. Pesquisa Em Psicologia - Anais eletrônicos, 21 - 23. 2016. Disponível em: < https://portalperiodicos.unoesc.edu.br/pp_ae/article/view/11914>. Acesso em: 29 ago. 2020.

BENEDITO, J. G. SÍNDROME DE BURNOUT EM ENFERMEIROS NA UNIDADE DE TERAPIA INTENSIVA: UMA REVISÃO NARRATIVA DA LITERATURA. Uma Revisão Narrativa da Literatura, Pernambuco, p.01-13, 2017.

CASTRO, E. K.; MASSOM, T.; DALAGASPERINA, P. Estresse Traumático Secundário em Psicólogos. Revista Psicologia e Saúde, v.10, n. 1, p.115-125, 23 mar. 2018. Universidade Católica Dom Bosco. Disponível em: <http://dx.doi.org/10.20435/pssa.v9i3.554>. Acesso em: 10 fev. 2020.

CENTRO DE REFERÊNCIA EM SAÚDE DO TRABALHADOR- CEREST. Biblioteca Virtual Em Saúde. 2015. Disponível em: <http:// bvsms.saude.gov.br/dicas-emsaude/1086-centro-de-referencia-em-saude-do-trabalhador-cerest>. Acesso em: 30 ago. 2020.

CEREST - APRESENTAÇÕES. POLÍTICA NACIONAL DE SAÚDE DO TRABALHADOR E DA TRABALHADORA. Vigilância em Saúde. Disponível em: <https://www.vs.saude.ms.gov.br/cerest/apresentacoes-cerest/>. Acesso em: 23 ago. 2020.

COFEN, Conselho Federal de Enfermagem. Resolução Cofen 633/2020. A Atuação dos profissionais de enfermagem no atendimento Pré-hospitalar (APH) móvel terrestre e Aquaviário, quer seja na assistência direta e na Central de fregulação das Urgências (CRU). Brasília, 24 de Març de 2020. Disponível em: <http://www.cofen.gov.br/resolucao-cofen-no-0633-

2020_78203.html\#: :text=Normatiza\%2C\%20\%E2\%80\%9Cad\%20referendum\%E2 \%80\%9D\%20do,Regula\%C3\%A7\%C3\%A30\%20das\%20Urg\%C3\%AAncias\%20(CR U)>. Acesso em 13 dez. 2020.

RC: 79470

Disponible: https://www.nucleodoconhecimento.com.br/salud/estres-traumaticosecundario 
COSTA, C. A. C.; COSTA, V. L. S.; ESTRESSE OCUPACIONAL EM TRABALHADORES DA SAÚDE: FATORES DE RISCO E AGRAVOS GERADOS NO AMBIENTE DE TRABALHO:X Semana de Iniciação Científica da Faculdade R. Sá. Pi. jun. 2016.

FABRI, J. M.G.; NORONHA, I.R.; OLIVEIRA, E.B.; KESTENBERG, C. C. F., HARBACHE, L. M. A.; NORONHA, I. R Estres se ocupacional em enfermeiros da pediatria: manifestações físicas e psicológicas. Rev baiana enferm. V. 32, 25070. 2018.

Disponível em:

ttps://portalseer.ufba.br/index.php/enfermagem/article/view/25070>. Acesso em: 30 ago. 2020.

FELLI, V. E. A; COSTA, T. F; BAPTISTA, P. C. P; GUIMARÃES, A. L. O; ANGINO, B.M. Exposição dos Trabalhadores de Enfermagem às Cargas de Trabalho e Suas consequências. Revista da Escola de Enfermagem da USP. 49. Pág. 98 - 105. 2015.

FREIRE, D. A. L; MARISA, A. E; Levantamento dos mecanismos de defesa dos profissionais de enfermagem frente à deterioração das condições de trabalho: Rev. Adm. Saúde Vol. 17, № 68, jul. - set. 2017. Acesso em: 19 fev. 2020.

FREITAS, R. J. M., et al. Estresse do enfermeiro no setor de urgência e emergência. Revista de Enfermagem UFPE on line- ISSN:1981-8963, v. 9, n. 10, p. 1476-1483, nov. 2015. ISSN 1981-8963. Disponível em: <https://periodicos.ufpe.br/revistas/revistaenfermagem/article/view/10861>. Acesso em: 07 fev. 2019.

GAMBARELLI, S. F; TAETS, G.G.C. C; Revisão A importância da empatia no cuidado de enfermagem na atenção primária à saúde. v. 17, n.4, 2018. Disponível em: <http://dx.doi.org/10.33233/eb.v17i4>. Acesso em: 02 mar. 2020.

FROTA, M. A. et al.; Mapeando a formação do enfermeiro no Brasil: desafios para atuação em cenários complexos e globalizados. Ciênc. saúde coletiva, Rio de 
Janeiro , v. 25, n. 1, p. 25-35, Jan. 2020 . Disponível em: $<$ http://www.scielo.br/scielo.php?script=sci_arttext\&pid=S1413$81232020000100025 \&$ Ing=en\&nrm=iso >. Acesso em: 15 dez. 2020.

GUIMARÃES, A. L. O; FELLI, V. E. A; Notificação de Problemas de Saúde em Trabalhadores de Enfermagem de Hospitais Universitários. Revista Brasileira de Enfermagem. Vol. 69. № 03 Brasília Maio/Junho 2016. <https://doi.org/10.1590/0034-7167.2016690313i>. Acesso em: 06 agos. 2020.

GOMES, J.; SILVA, A. S.; BERGAMINI, G. B. SAÚDE E QUALIDADE DE VIDA: INFLUÊNCIA DO STRESS NO AMBIENTE DE TRABALHO: Revista Científica da Faculdade De Educação E Meio Ambiente FAEMA.v.8, n.2,207 - 220, Ariquemes, 2017. Disponível em: <https://doi.org/10.31072/rcf.v8i2.547>. Acesso em: 18 fev. 2020.

HIPÓLITO, M.C.V.; MASSON, V.A.; MONTEIRO, M. I.; GUTIERREZ, G. L. Qualidade de Vida no Trabalho: Avaliação de Estudos de Intervenção. Revista brasileira de Enfermagem [Internet]. 2016; 70 (1): 178-86. Acesso em: 21 setembro, 2020.

LOPES, E. R.; SOUZA, J. E. O. Conflitos na equipe de enfermagem: um desafio na gestão do enfermeiro.: Única Cadernos Acadêmicos. Contagem - MG, p.01-10, 2015.

em: $<$ http://co.unicaen.com.br:89/periodicos/index.php/UNICA/article/view/10>. Acesso em: 03 fev. 2020.

LORENZO, R. A; GUERRERO, A. A. E.; Afetações psicológicas em casos pessoais primários: ¿Trastorno por períodos pós-traumáticos ou períodos traumáticos secundários? Preocupações psicológicas nos socorristas: transtorno de estresse póstraumático ou estresse traumático secundário? Revista Puertorriqueña de Psicología, 28 (2), 252-265. 2017.

MORAES FILHO, I. M.; Estresse Ocupacional no Trabalho em Enfermagem no Brasil: Uma Revisão Integrativa: Revista Brasileira em Promoção da Saúde. 
V.29, n.3, p.447-454, ago. 2016. Disponível em: <https://www.redalyc.org/articulo.oa?id=40849134018>. Acesso em: 19 fev. 2020.

MUFATO, L. F; GAIVA, M. A. M; EMPATIA EM SAÚDE: REVISÃO INTEGRATIVA. Revista de Enfermagem do Centro Oeste Mineiro. 2019;9: e2884. Disponível em: <http://dx.doi.org/10.19175/recom.v9i0.2884>. Acesso em: 21 fev. 2020.

OLIVEIRA, E. B. et a. Estresse ocupacional e burnout em enfermeiros de um serviço de emergência: a organização do trabalho [Occupational stress and burnout in nurses of an emergency service: the organization of work]. Revista Enfermagem UERJ, [S.I.], v. 25, p. e28842, jun. 2017. ISSN 0104-3552. Disponível em: <https://www.epublicacoes.uerj.br/index.php/enfermagemuerj/article/view/28842/22382>. Acesso em: 25 fev. 2020.

Organização Mundial da Saúde. Classificação Estatística Internacional de Doenças e Problemas Relacionados à Saúde: CID-10 Décima revisão. Trad. Do Centro Colaborador da OMS para a Classificação de Doenças em Português. 3 ed. São Paulo: EDUSP; 1996.

PRETO, V. A.; PEDRÃO, L J. O estresse entre enfermeiros que atuam em Unidade de Terapia Intensiva. Revista da Escola de Enfermagem da Usp, v. 43, n. 4, p.841848, dez. 2016 FapUNIFESP (SciELO). Disponível em: <http://dx.doi.org/10.1590/s0080-62342009000400015>. Acesso em: 30 jan. 2020.

RISSARDO, M. P.; GASPARINO, R. C. Exaustão emocional em enfermeiros de um hospital público: Esc. Anna Nery, Rio de Janeiro, v.17, n.1, p.128132, mar. 2013. Available from

<http://www.scielo.br/scielo.php?script=sci_arttext\&pid=S1414-

$81452013000100018 \&$ Ing=en\&nrm=iso $>$. Acesso em: 17 fev. 2019.

RODRIGUES, C. P. Estresse e qualidade de vida em técnicos e auxiliares de enfermagem em instituições de longa permanência para idosos.: O Mundo Saúde, n. 2, v. 40, São Paulo, p.180-188, 2017. 
RUBACK, S.P; TAVARES, J.M.A.B; LINS, S.M.S.B; et al. Estresse e Síndrome de Burnout em Profissionais de Enfermagem que Atuam na Nefrologia: Uma Revisão Integrativa. Rev Fund Care Online. 2018 jul. /Set.; 10(3):889-899. Disponível em: <http://dx.doi.org/10.9789/2175-5361.2018.v10i3.889-899>. Acesso em: 10 fev. 2020.

RUIZ, A. L; ANGELES, E. A. G. Afectaciones Psicológicas Em Personal De Primeira Respuesta: Transtorno Por Estrés Postraumático O Estrés Traumático Seundário? Revista Puertorriquena de Psicologia. V. 28. № 2. Pág. 252 -265. Julho - dezembro 2017.

SANCHEZ, H. M. et al. Impacto da saúde na qualidade de vida e trabalho de docentes universitários de diferentes áreas de conhecimento. Ciência \& Saúde Coletiva [online]. v. 24, n. 11, 2019. Acessado 16 setembro 2020, pp. 4111-4123. Disponível em: <https://doi.org/10.1590/1413-812320182411.28712017>.

SANTANA, J. R.; RODRIGUES, C. C.; PEREIRA, S. F.; ANÁLISE DE CONTEÚDO DA OBRA FADIGA POR COMPAIXÃO, DE KENNYSTON LAGO E WANDERLEY CODO: SOB O OLHAR DA ENFERMAGEM: Revista Pesquisa E Ação, 3(1); (2017, junho

Recuperado de <https://revistas.brazcubas.br/index.php/pesquisa/article/view/268>. Acesso em: 22 fev. 2020.

SAVIETO, R. M; RIBEIRO; LEÃO, E. R. Assistência em Enfermagem e Jean Watson: Uma reflexão sobre a empatia. Escola Anna Nery Revista de Enfermagem 2016, vol.20, n.1, pp.198-202. ISSN 1414-8145. 2020 Disponível em: <https://doi.org/10.5935/1414-8145.20160026>. Acesso em: 02 mar. 2020.

SBARDELLOTO, G et al. Processamento Cognitivo no Transtorno de Estresse PósTraumático: Um Estudo Teórico. Interação em Psicologia, Curitiba, v. 16, n. 2, dez. 2012. ISSN 1981-8076. Disponível em: <https://revistas.ufpr.br/psicologia/article/view/18934>. Acesso em: 20 fev. 2020. 
SILVA, D. P; SANTOS, N. R. O; NASCIMENTO, L.K.A.S; Fatores Que Influenciam o Estresse Ocupacional Na enfermagem: Revista Cultural e Científica do UNIFACEX. v. 14, n. 2, outubro de 2016.

SILVA, R. M; GOULART, C.T; GUIDO, L.A. Evolução histórica do conceito de estresse. Rev. Cient. Sena Aires. 2018; 7 (2): p. 148-156. Acesso em: 04 fev. 2020.

TEIXEIRA, G.S; SILVEIRA, R.C.P; MININELM, V.A; MORAES, J.T; RIBEIRO, I.K.S. Qualidade de vida no trabalho e estresse ocupacional da enfermagem em unidade de pronto atendimento. Texto Contexto Enferm 2019, 28: e20180298. Disponível em: <http://dx.doi.org/10.1590/1980-265X-TCE-2018-0298>. Acesso em: 27 Jan. 2020.

TEREZAM, R.; REIS-QUEIROZ, J; HOGA, L. A. K. The importance of empathy in health and nursing care. Revista Brasileira de Enfermagem, v. 70, n. 3, p.669-670, jun. 2017. FapUNIFESP (SciELO). Disponível em: <http://dx.doi.org/10.1590/00347167-2016-0032>. Acesso em: 04 fev. 2020.

TRETTENE, A. S., et al. Estresse em profissionais de enfermagem atuantes em Unidades de Pronto Atendimento. Bol. - Acad. Paul. Psicol., São Paulo, v. 36, n. 91, p. 243-261, jul. 2016. Disponível em <http://pepsic.bvsalud.org/scielo.php?script=sci_arttext\&pid=S1415711 X2016000200002\&lng=pt\&nrm=iso >. Acesso em: 04 fev. 2020.

VALENTE, N. L. M; MELLO, M. F.; FIKS, J. P. Transtorno de Estresse pós-traumático. In: MARI, Jair de Jesus. Psiquiatria na Prática Clínica. Tamboré: Manole. p. 315326, Cap. 18, 2013.

VAZATA, D.; NEZI, T. C. O ESTRESSE E A PROFISSÃO DE ENFERMAGEM. Pesquisa em Psicologia - anais eletrônicos, 30 out. 2015. Disponível em

https://portalperiodicos.unoesc.edu.br/pp_ae/article/view/8609> Acesso em 28 jan. 2020. 
VERSA, G. L. G. S.; MURASSAKI, A. C. Y.; INOUE, K. C.; MELO, W. A.; FALLER, J. W.; MATSUDA, L. M. Estresse ocupacional: avaliação de enfermeiros intensivistas que atuam no período noturno. Rev Gaúcha Enferm. 33, p.78-85, jun. 2012.

VIEIRA, S.S.; AZEVEDO, I. R. P.; CASAGRANDE, J. L. Pesquisa Bibliográfica, Pesquisa Bibliométrica, Artigo de Revisão e Ensaio Teórico em Administração e Contabilidade. Administração: Ensino e Pesquisa. Vol.19, n. 2, 2018. Maio agosto, pg 308-339 Rio de Janeiro. Disponível em: <https://doi.org/10.13058/raep.2018.v19n2.970> Acesso em: 10 fev. 2020.

Recibido: Diciembre de 2020.

Aprobado: Enero de 2021. 